\title{
ECOLOGIE DES CRUSTACÉS BENTHIQUES DU LAC DE PORT-BIELH (PYRENÉES CENTRALES) : I. REPARTITION
}

\author{
par J. REY ${ }^{1}$ et B. DUPIN ${ }^{1}$.
}

\begin{abstract}
Des populations benthiques de Cladocères, Copépodes et Ostracodes ont été suivies dans un lac de haute montagne pendant la période où les eaux étaient dégagées des glaces. Quelques espèces dominantes constituent l'essentiel de la biomasse en crustacés. Deux communautés sont distinguées, correspondant aux zones littorale $(0-7 \mathrm{~m})$ et littori-profonde $(7-19 \mathrm{~m})$. La nature du substrat, la température et la teneur en oxygène sont mises en relation avec la distribution des espèces. Les conditions thermiques semblent jouer un rôle primordial tant dans la répartition que dans l'évolution des populations.
\end{abstract}

\section{Ecology of benthic crustacea in Lake Port-Bielh (Central Pyrenees) I. Distribution.}

Populations of benthic Cladocera, Copepoda and Ostracoda have been studied in a high mountain lake during the ice-free period. Several dominant species form most of crustacean biomass. Two communities are recognised, and correspond to the littoral zone $(0-7 \mathrm{~m})$ and the littoral-profundal zone $(7-19 \mathrm{~m})$. The nature of the substratum, the temperature and the oxygen content are examined in relation to the distribution of species. The thermal conditioss seem to play a basic role in the distribution and development of the populations.

\section{INTRODUCTION}

D'une manière générale les écosystèmes aquatiques de haute montagne, en raison de leurs conditions extrêmes de milieu, sont caractérisés par une relative simplicité de leurs communautés biotiques. C'est pourquoi le lac de Port-Bielh, lac naturel situé à 2285 mètres, a été choisi afin d'étudier la structure et le fonctionnement d'un lac d'altitude et préciser sa productivité.

Répondant à cet objectif un certain nombre de travaux ont été réalisés : étude morphométrique et physico-chimique (Capblancq

1. Laboratoire d'Hydrobiologie, Université Paul-Sabatier, 118, route de Narbonne, 31077 Toulouse Cedex. 
et Laville 1968), phytoplancton et production primaire (Capblancq 1972), populations macrobenthiques (Laville $1971 \mathrm{a}, 1971 \mathrm{~b}$; Giani et Laville 1973; Laville et Giani, sous presse), zooplancton (Rey et Capblancq, en préparation).

Ce travail constitue une étude préliminaire du microbenthos et envisage plus particulièrement la structure et l'évolution des populations benthiques de Cladocères, Copépodes et Ostracodes. Les cycles biologiques des principales espèces, données de base à toute estimation de productivité, seront abordés dans un travail ultérieur.

\section{1. - LE MILIEU; SES CARACTERES PHYSICO-CHIMIQUES}

Les données détaillées concernant les caractères morphométriques et physico-chimiques ayant déjà été publiées (Capblancq et Laville 1968) nous renvoyons à ce travail et rappelons simplement ici les caractéristiques essentielles.

Situé dans le massif du Néouvielle (Pyrénées Centrales), le lac de PortBielh, lac d'origine glaciaire, est essentiellement alimenté par les eaux de fonte des neiges et, pendant l'été, par quelques sources de faible débit qui coulent dans sa partie orientale.

D'une superficie de 16,5 ha, le lac atteint une profondeur maximale de $19 \mathrm{~m}$. La topographie du fond permet de distinguer deux grandes zones qui correspondent aux zones «littorale» et «littori-profonde» définies par Capblancq et Laville (1972). La zone «littorale», de $0 \mathrm{~m}$ à $7 \mathrm{~m}$ de profondeur, formée d'éboulis granitiques entre lesquels se sont accumulés sables, vase et débris ogganiques exogènes, représente $37 \%$ de la surface totale. Elle est pratiquement dépourvue de végétation fixée sauf à l'ouest du déversoir du lac où il se constitue des plages envasées favorisant le développement d'Isoetes brochoni.

La zone «littori-profonde» comprise entre $7 \mathrm{~m}$ et $19 \mathrm{~m}$ de profondeur s'oppose à la précédente par l'homogénéité du substrat. Recouverte d'une couche de vase uniforme, elle présente une importante prairie de Nitella flexilis qui disparaît progressivement au-delà de $16 \mathrm{~m}$.

- Régime thermique: le lac est gelé en moyenne de novembre à juin, période pendant laquelle il est recouvert d'une épaisse couche de glace pouvant atteindre 1,50 à $3 \mathrm{~m}$. De type dimictique, le lac présente au moment du dégel une brève période d'isothermie $\left(0^{\circ} \mathrm{C}\right.$ à $\left.4^{\circ} \mathrm{C}\right)$ suivie d'une stratification thermique s'étendant environ jusqu'à la mi-septembre. Une longue isothermie automnale de $10^{\circ} \mathrm{C}$ à $13^{\circ} \mathrm{C}$ s'installe ensuite jusqu'à la fin novembre, moment où les eaux sont reprises par le gel. En 1971, année où se situent ces observations, le dégel s'est produit dans la dernière décade de juin. La température maximale enregistrée a été de $14^{\circ} \mathrm{C}$, au niveau de la zone. "littorale»

- Oxygène : en été, les eaux sont généralement saturées ou sursaturées. I.es taux d'oxygène les plus élevés apparaissent en août, en relation avec l'intensité photosynthétique du phytoplancton et des Nitella flexilis. Durant l'isothermie automnale, les valeurs sont proches de la saturation. En hiver, on constate un important déficit en profondeur. Les eaux restent sous-saturées pendant la circulation de printemps, la diffusion et la photosynthèse ne parvenant pas à combler le déficit hivernal.

- pH et gaz carbonique : toujours basiques, les $\mathrm{pH}$ varient entre 7,25 et 9,52. Ils augmentent au couss de l'été du fait de la diminution du CO2 libre. 
Cette alcalinisation, surtout nette au-delà de $10 \mathrm{~m}$ de profondeur dans la zone des Nitella, correspond aux concentrations maximales en 02 dissous. L'isothermie automnale entraîne l'égalisation des $\mathrm{pH}$ dans toute la colonne d'eau. En hiver, les valeurs tendent vers la neutralité.

- Eléments dissous : les eaux du lac de Port-Bielh sont très faiblement minéralisées. Elles se caractérisent par un pourcentage élevé des teneurs en carbonates et par de très faibles quantités de nitrates, phosphates, silice.

- Propriétés optiques : la transparence des eaux est élevée. Profondeur de visibilité : 13,5 en moyenne, avec des limites de visibilité variant de $9,5 \mathrm{~m}$ à $16,5 \mathrm{~m}$. Des mesures effectuées en 1970 et 1971 à l'aide d'une cellule photoélectrique immergée aux différentes profondeurs, permettent d'évaluer la transmission moyenne à $85,5 \%$.

\section{II. - LE PEUPLEMENT EN CRUSTACÉS}

\section{Méthodes d'échantillonnage.}

Les données qui suivent sont basées sur l'examen de 15 séries de prélèvements benthiques effectués au cours de l'élé 1971, environ lous les 10 jours du 22 juin (début du dégel du lac) au 5 novembre. Chacune des séries de prélèvements a été réalisée aux 7 profondeurs suivantes : $1 \mathrm{~m}, 3 \mathrm{~m}, 5 \mathrm{~m}, 7 \mathrm{~m}, 9 \mathrm{~m}, 12 \mathrm{~m}$ et $16 \mathrm{~m}$.

L'hétérogénéité du substrat, constitué au moins dans la partie littorale par une zone d'éboulis granitiques, nous a conduits à utiliser une drague de type Petersen. L'ouverture réduite de cet appareil $\left(0,020 \mathrm{~m}^{2}\right)$ permet de prélever entre les blocs rocheux, au niveau des zones d'accumulation de vase souvent exiguës On connaît les limites de fiabilité de cette drague quant à l'obtention de données quantitatives : en effet, comme le soulignent Edmonson et Winberg (1971), cet appareil opère de telle sorte que surface et profondeur de prise de sédiments sont imprécises. Toutefois les échantillonnages ayant été, lors de chaque prélèvement, réalisés dans des conditions rigoureusement identiques, l'utilisation de cet appareil fournit des données comparables entre elles. Ces prélèvements de vase étaient ensuite concentrés sur filet fin (vide de maille de $120 \mu$ ), formolés sur place et triés au laboratoire sous loupe binoculaire.

Par ailleurs, nous avons obtenu des données complémentaires à partir de l'examen de pièges à émergence (cônes de gaze de $50 \mathrm{~cm}$ de hauteur surmontés d'un flacon de $500 \mathrm{ml}$ ), installés en différents points du lac, pour la capture des imagos de Chironomides (Laville 1971 a).

\section{Liste faunistique.}

Au total 20 espèces de Crustacés appartenant aux Cladocères, Copépodes, Ostracodes ont été dénombrées. 
- Cladocères

* Daphniidae

Simocephalus vetulus (O. F. Muller)

* Macrothricidae

Ilyocryptus sordidus (Lieven)

Macrothrix hirsuticornis Norman et Brady

* Chydoridae

Eurycercus lamellatus (O. F. Muller)

Acroperus harpae Baird

Alona intermedia Sars

Alona affinis Leydig

Alona quadrangularis (O. F. Muller)

Alona guttata Sars

Chydorus sphaericus (O. F. Muller)

Chydorus gibbus Lilljeborg

- Copépodes

* Cyclopides

Macrocyclops fuscus (Jurine)

Eucyclops serrulatus (Fischer)

Paracyclops fimbriatus (Fischer)

Cyclops strenuus Fischer

* Harpacticides

Canthocamptus staphylinus (Jurine)

Attheyella crassa (Sars)

Bryocamptus zschokkei (Schmeil)

- Ostracodes ${ }^{1}$

* Cypridae

Cypria cf. lacustris Sars

Candona cf. neglecta Sars

La plupart des espèces récoltées sont des formes à vaste répartition constituant une faune assez banale, au sein de laquelle on note cependant la présence de quelques éléments boréo-alpins tels Macrothrix hirsuticornis, Alona intermedia et Bryocamptus zschokkei.

\section{Répartition et évolution saisonnière du peuplement.}

Répartition bathymétrique : la majorité des espèces montre une répartition bathymétrique assez nette. Nous avons représenté (fig. 1) l'abondance relative des individus de chaque espèce aux

1. Nous remercions vivement le $\mathrm{D}^{r}$ Dan Danielopol (Institut de Spéologie, Bucarest) qui a bien voulu assurer la détermination de ce matériel. 
différentes profondeurs, calculée sur l'ensemble des prélèvements réalisés au cours de l'été (les espèces récoltées en nombre trop faible n'ont pas été prises en considération).

L'examen de ce diagramme permet de distinguer dans ce peuplement de Crustacés benthiques, deux groupements d'espèces correspondant aux deux biotopes déjà définis :

- le premier groupement, correspondant à la zone «littorale», est représenté par 6 espèces; 3 d'entre elles : Attheyella crassa, Bryocamptus zachokkei, Paracyclops fimbriatus sont strictement littorales et peuvent être considérées commes espèces caractéristiques du groupement. Elles sont associées à Eucyclops serrulatus, Alona affinis et Alona quadrangularis, espèces plus eurybathes mais dont le maximum de développement se situe dans la zone « littorale »;

— le deuxième groupement, correspondant à la zone « littoriprofonde » est composé de 11 espèces. Parmi celles-ci Eurycercus lamellatus, Acroperus harpae, Simocephalus vetulus, Canthocamptus staphylinus, Cypria cf. lacustris et Candona cf. neglecta, strictement inféodées à la zone profonde du lac, constituent les espèces caractéristiques du groupement. Elles s'associent à Alona affinis, Alona quadrangularis, Ilyocryptus sordidus, Chydorus sphaericus et Cyclops strenuus; ces deux dernières colonisent toute la colonne d'eau mais montrent cependant un preferendum pour la zone «littori-profonde».

Evolution saisonnière du peuplement : la structure et l'évolution du peuplement ont été résumées dans deux séries de diagrammes en cercles figurant l'abondance relative de chaque espèce. Ces deux séries correspondent respectivement au peuplement des zones 《littorale » et «littori-profonde ».

— En zone « littorale» (fig. 2), l'essentiel du peuplement est constitué par 3 espèces : Attheyella crassa, Paracyclops fimbriatus et Alona affinis qui représentent de 78,5\% à $97,9 \%$ (valeurs extrêmes) des Crustacés benthiques.

Ces trois espèces dominantes se relayent dans le temps en fonction de leur cycle évolutif. En début de saison, l'Harpacticide et le Cyclopide, alors en période de reproduction, représentent jusqu’à $75 \%$ du peuplement. Cette dominance s'exerce jusque vers la fin août.

Alona affinis devient ensuite prépondérante pendant tout le mois de septembre, époque où sa population atteint le maximum de densité. Son abondance relative varie de 50 à $67 \%$ du peuplement littoral. Au début du mois d'octobre, les trois espèces sont représentées de façon à peu près identique.

A l'entrée de l'hiver, le 5 novembre, Attheyella crassa et Paracyclops fimbriatus constituent la presque totalité du peuplement 


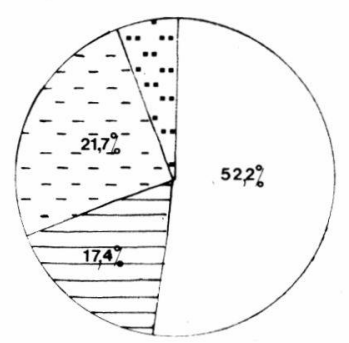

3 Juillet

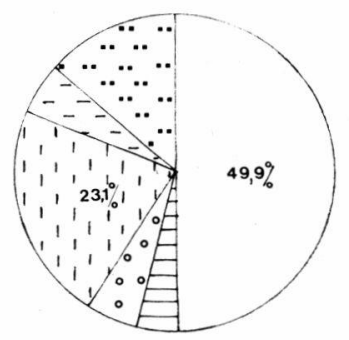

24 Aout

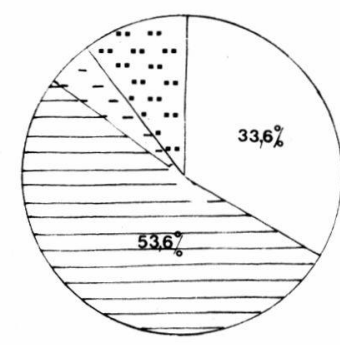

21 Juillet

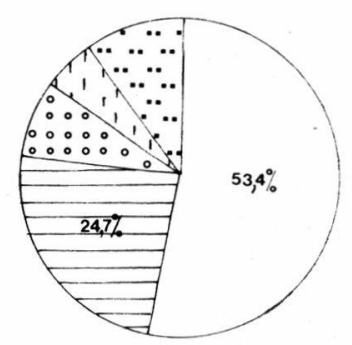

4 Aout

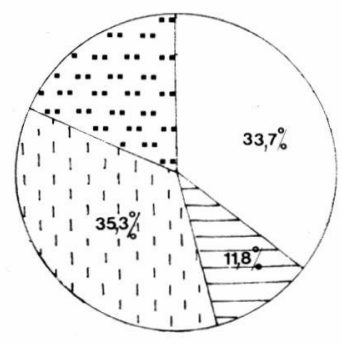

8 Septembre

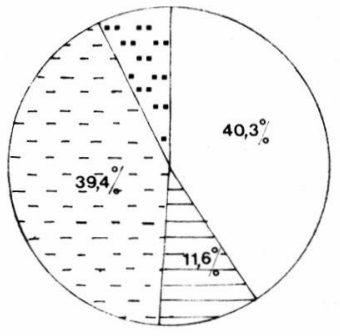

28 Septembre

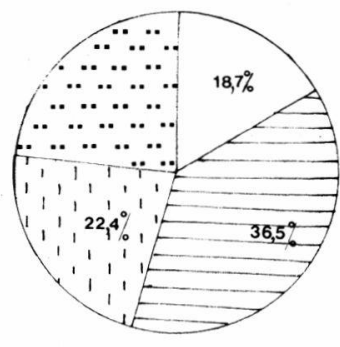

12 Octobre

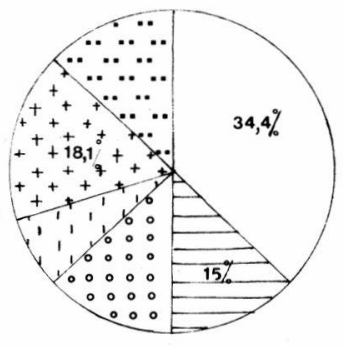

5 Novembre

C. staphylinus

E. lamellatus Autres espèces

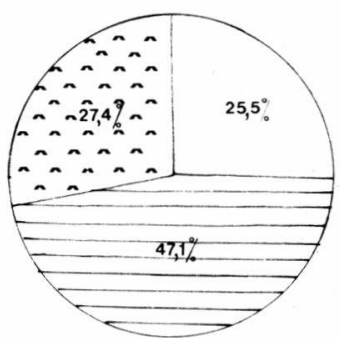

29 Mars

\section{A. affinis}

C. sphoericus

Fig. 2. - Evolution du peuplement de Crustacés benthiques dans la zone «littorale» du lác de Port-Bielh, au cours de l'année 1971. 
$(97,9 \%)$. Alona affinis a disparu et n'est plus représentée que par des éphippies.

Alona quadrangularis, Ilyocryptus sordidus, Bryocamptus zschokkei sont présentes dans plus de la moitié des prélèvements, mais en faible nombre. Les autres espèces (Alona intermedia, Cyclops strenuus, Chydorus sphaericus, Macrocyclops fuscus) récoltées en très petite quantité dans moins de $25 \%$ des prélèvements, peuvent être considérées comme accidentelles ou rares.

- En zone «littori-profonde» (fig. 3) les espèces dominantes sont Cypria ef. lacustris, Canthocamptus staphylinus, Eurycercus lamellatus et Alona affinis. Elles représentent jusqu'à $95 \%$ de la totalité du peuplement.

L'Ostracode Cypria cf. lacustris est l'espèce dominante jusqu'à la fin août (valeurs atteignant $53,4 \%$ ). En septembre, période où les populations de Cladocères atteignent leur densité optimale, Eurycercus lamellatus au début du mois et Alona affinis en fin de mois, représentent la majeure partie du peuplement.

En octobre, l'espèce dominante est Canthocamptus staphylinus dont la nouvelle génération est issue de la reproduction de juillet. A l'entrée de l'hiver, Canthocamptus staphylinus et Cypria cf. lacustris constituent la moitié du peuplement. Toutefois il faut noter à cette date, l'importance relative des deux Cladocères Chydorus sphaericus $(18,1 \%)$ et Acroperus harpae $(14,7 \%)$.

A côté de ces espèces dominantes, Acroperus harpae, Simocephalus vetulus, Chydorus sphaericus, Candona ef. neglecta, Ilyocryptus sordidus, Cyclops strenuus ont été trouvées en faible nombre mais dans plus de la moitié des prélèvements.

Eucyclops serrulatus, Macrocyclops fuscus, Alona quadrangularis sont les espèces rares de cette zone «littori-profonde».

Quelques prélèvements d'hiver ont permis d'observer la composition du peuplement durant cette période. Le 29 mars 1971, à 16 mètres de profondeur, 3 espèces constituent le peuplement benthique du lac : Canthocamptus staphylinus (46\%), Cyclops strenuus et Cypria cf. lacustris.

\section{DISCUSSION}

Si la majorité des espèces présente une assez nette répartition bathymétrique, la comparaison de la faune capturée dans les pièges à émergences de Chironomides avec la faune provenant des prélèvements réalisés à la drague a permis de préciser la plus ou moins grande dépendance des espèces vis-à-vis du substrat.

Ainsi, parmi les différents Crustacés qui ont été récoltés à la drague, un certain nombre d'entre eux : les trois Harpacticides, 


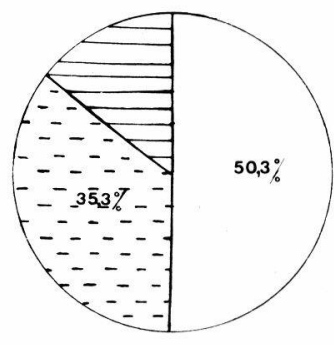

22 Juin

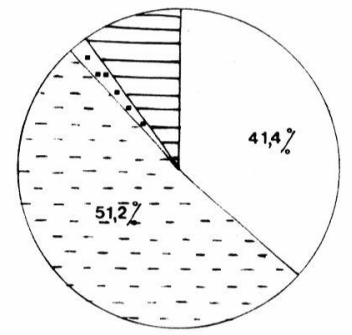

3 Juillet

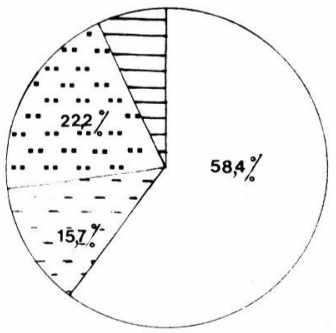

21 Juillet

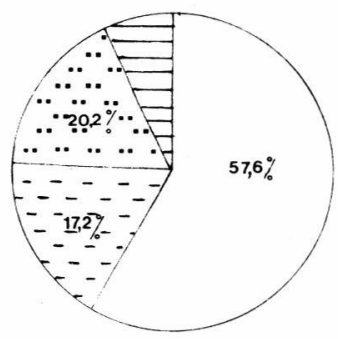

4 Aout

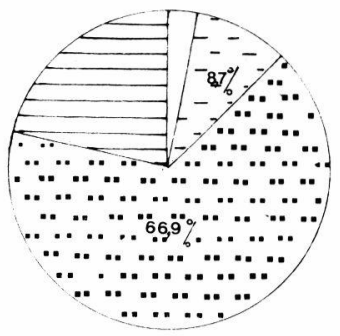

28 Septembie

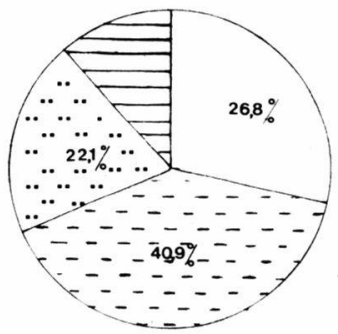

24 Aout

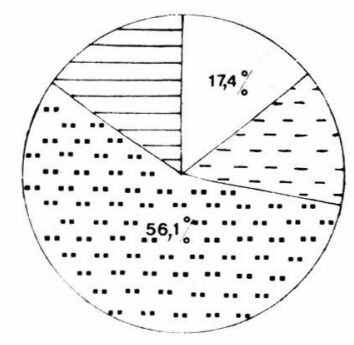

8 Septembre

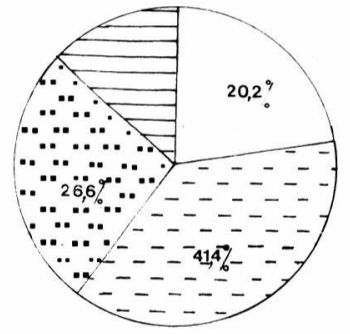

12 Octobre

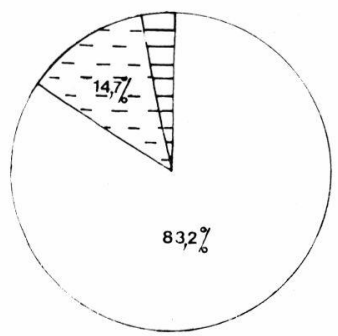

5 Novembre
A. affinis Autres especes

Fig. 3. - Evolution du peuplement de Crustacés benthiques dans la zone «littori-profonde» du lac de Port-Bielh, au cours de l'année 1971. 
les deux Ostracodes et les Cladocères Simocephalus vetulus et Ilyocryptus sordidus, n'ont jamais été recueillis dans les pièges. D'autres comme Alona affinis, A. quadrangularis, Acroperus harpae, Eurycercus lamellatus étaient présents dans les flacons de capture mais de façon accidentelle et toujours en petit nombre d'individus. Ces différentes espèces se révèlent étroitement cantonnées au contact du substrat.

Par contre, parmi la faune capturée dans ces pièges, nous avons pu observer, à côté de formes euplanctoniques telles que Mixodiaptomus laciniatus, de nombreux Cyclops strenuus, Macrocyclops fuscus, Eucyclops serrulatus et Chydorus sphaericus.

Ces espèces paraissent donc moins inféodées au substrat et capables d'évoluer dans une zone assez large à partir du fond. Il s'agit là d'espèces douées d'une grande mobilité et dont le mode de nutrition (Fryer 1957) algivore (Eucyclops serrulatus) ou prédateur (Cyclops strenuus, Macrocyclops fuscus), exige un certain déplacement. Il faut souligner l'importance de ce déplacement puisque certains pièges se situent, au niveau de la zone "littoriprofonde ", à 8-10 mètres du fond. Etant donné que ces observations portent sur des relevés effectués toutes les semaines, on peut se demander si ce déplacement est un phénomène constant ou s'il est soumis à certains rythmes nycthéméraux.

Quant aux espèces strictement benthiques, leur répartition est essentiellement liée à la nature du substrat. La localisation de Simocephalus vetulus, espèce phytophile, au niveau des Nitella et celle d'llyocryptus sordidus au contact de la vase, confirment l'écologie de ces espèces. De même Eurycercus lamellatus, forme très exigeante du point de vue teneur en oxygène dissous comme l'a montré expérimentalement Pacaud (1939), trouve au voisinage des Nitella des conditions optimales d'oxygénation. Une répartition analogue avait déjà été observée (Rey 1968) tant en profondeur qu'en zone littorale. Dans les deux cas, le milieu était caractérisé par des taux d'oxygène très élevés. Il semble que pour cette espèce le facteur oxygénation soit primordial. Ces observations rappellent l'opinion de Macan (1970) selon laquelle la faune benthique reflète essentiellement les conditions qui règnent au contact du substrat, ne constituant de ce fait qu'un mauvais indicateur des caractères généraux du lac.

Si l'on examine l'évolution saisonnière du peuplement, on constate que les espèces caractéristiques constituent, tant dans la zone "littorale " que dans la zone "littori-profonde», la majorité des espèces dominantes : Attheyella crassa, Paracyclops fimbriatus auxquelles s'ajoute Alona affinis représentent jusqu'à $95 \%$ du peuplement littoral; Cypria cf. lacustris, Canthocamptus staphyli- 

A. affinis
C. lacustris
A. crassa
P. fimbriatus
C. staphylinus
E. lamellatus

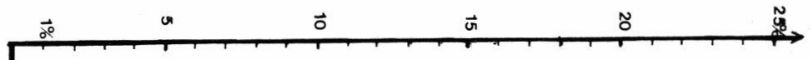
A. harpae
I. sordidus
C. sphaericus
A. quadrangularis
E. serrulatus
C. neglecta

B. zschokkei

C. strenuus

S. vetulus

M. fuscus

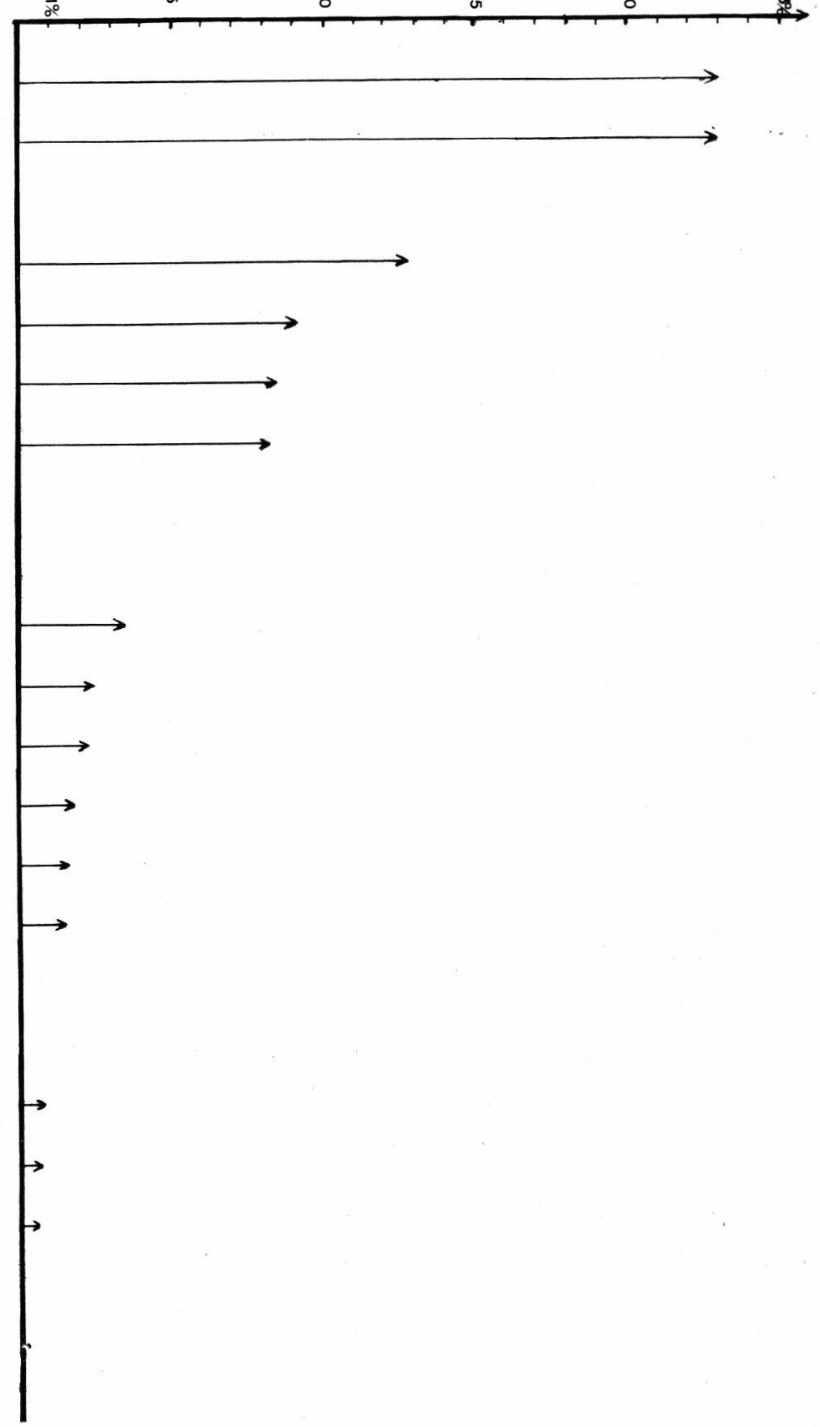

Fig. 4. - Abondances relatives moyennes des principales espèces de Crustacés benthiques sur l'ensemble du lac de Port-Bielh.

nus, Eurycercus lamellatus représentent avec Alona affinis et Acroperus harpae $85 \%$ du peuplément de profondeur. 
En ce qui concerne l'abondance relative des espèces sur l'ensemble du lac (fig. 4), Alona affinis et Cypria cf. lacustris constituent à elles deux presque $50 \%$ du peuplement total.

Il faut noter la pauvreté en espèces et en individus de la zone comprise entre $5 \mathrm{~m}$ et $7 \mathrm{~m}$, zone de transition entre la partie «littorale» et la partie profonde du lac.

Nous avons tenté de caractériser ce peuplement benthique de Crustacés aux différentes dates de prélèvement, par le rapport $\sigma / \alpha$ (Bonnet 1964) où $\sigma$ représente l'écart-type de la distribution des abondances relatives et $\alpha$ l'indice de diversité spécifique (tableau ci-dessous).

\begin{tabular}{|c|c|c|c|c|c|c|c|c|c|c|}
\hline Dates & & 22.6 & 3.7 & 21.7 & 4.8 & 24.8 & 8.9 & 28.9 & 12.10 & 5.11 \\
\hline N兽 & $\begin{array}{c}\sigma \\
\alpha \\
\sigma / \alpha\end{array}$ & $\begin{array}{r}11,5 \\
1,7 \\
6,8\end{array}$ & $\begin{array}{c}6,4 \\
1,4 \\
4,5\end{array}$ & $\begin{array}{c}6,7 \\
1,8 \\
3,6 \\
-\end{array}$ & $\begin{array}{l}6,8 \\
1,7 \\
4,0 \\
-\end{array}$ & $\begin{array}{c}6,2 \\
1,6 \\
3,3 \\
-\end{array}$ & $\begin{array}{l}7,7 \\
1,2 \\
6,4\end{array}$ & $\begin{array}{l}7,4 \\
1,4 \\
5,3\end{array}$ & $\begin{array}{l}6,2 \\
1,3 \\
4,8\end{array}$ & $\begin{array}{r}13,7 \\
1,2 \\
11,4\end{array}$ \\
\hline 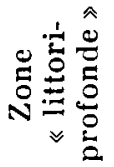 & $\begin{array}{c}\sigma \\
\alpha \\
\sigma / \alpha\end{array}$ & & $\begin{array}{l}8,6 \\
1,4 \\
6,1\end{array}$ & $\begin{array}{r}11,3 \\
1,3 \\
8,7\end{array}$ & $\begin{array}{r}5,5 \\
1,9 \\
2,9 \\
-\end{array}$ & $\begin{array}{l}4,6 \\
1,6 \\
2,8 \\
-\end{array}$ & $\begin{array}{r}4,1 \\
1,9 \\
2,1\end{array}$ & $\begin{array}{l}4,9 \\
1,8 \\
2,7 \\
-\end{array}$ & $\begin{array}{l}3,4 \\
2,0 \\
1,7 \\
-\end{array}$ & $\begin{array}{l}4,1 \\
1,3 \\
3,0\end{array}$ \\
\hline
\end{tabular}

L'évolution saisonnière de ce rapport traduit la faible diversité spécifique des deux milieux et la dominance de quelques espèces au début et à la fin de l'été. On retrouve là une caractéristique générale du peuplement des lacs de montagne où la colonisation est assurée par un nombre relativement restreint d'espèces et par la dominance de certaines d'entre elles.

Le développement des populations de Cladocères en août et septembre entraîne une diversification de la faune et une diminution de la valeur de ce rapport (diversification plus marquée au niveau de la zone profonde).

Si l'on compare les valeurs au cours de l'été du rapport $\sigma / \alpha$ dans les deux zones, le décalage des valeurs minimales se situe en début de saison (juillet-août) dans la zone «littorale et en fin de saison (août-septembre-octobre) dans la zone profonde. Ce décalage dans le temps peut être mis en relation avec les conditions thermiques des deux milieux (fig. 5). En zone «littorale», dès le dégel, les températures subissent une hausse brutale, passant de $0-1{ }^{\circ} \mathrm{C}$ à plus de $10^{\circ} \mathrm{C}$ en moins de dix jours. Les températures maximales sont atteintes en août. Les valeurs chutent à partir d'octobre, lors du gel du lac. En zone «littori-profonde», la 
hausse des températures après le dégel est beaucoup plus lente : d'environ $4{ }^{\circ} \mathrm{C}$ au moment du dégel, les eaux s'élèvent à $10{ }^{\circ} \mathrm{C}$ un

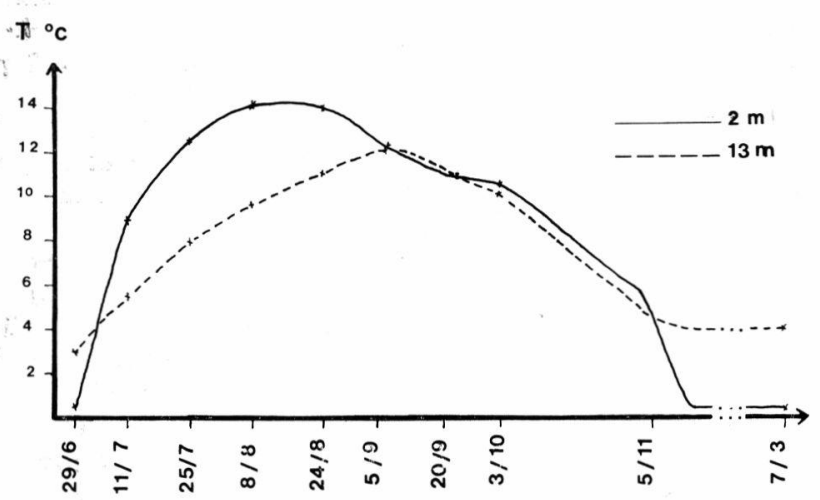

FIg. 5. - Evolution comparée des températures dans le lac de Port-Bielh, à $2 \mathrm{~m}$ et à $13 \mathrm{~m}$ de profondeur, en 1971 .

mois et demi plus tard (début d'août). Les températures maximales sont atteintes en septembre et ne dépassent pas $11{ }^{\circ} \mathrm{C}$. A l'entrée de l'hiver, les valeurs chutent progressivement jusqu'à se stabiliser aux environs de $4{ }^{\circ} \mathrm{C}$ (novembre) alors qu'en zone «littorale», à la même période, la température se rapproche de $0{ }^{\circ} \mathrm{C}$.

Les eaux littorales se réchauffent plus rapidement et atteignent leur température maximale un mois plus tôt que les couches profondes. Il semble donc bien exister une correspondance entre l'évolution thermique du milieu et les paramètres du peuplement.

Les conditions thermiques de la zone «littorale»favorisent un épanouissement des espèces beaucoup plus précoce qu'en zone profonde. En revanche, la chute brutale des valeurs tendant vers le zéro entraîne très vite, en fin de saison, la disparition des populations littorales.

\section{TRAVAUX GITÉS}

Bonnet (L.). 1964. - Le peuplement thécamoebien des sols. Rev. Ecol. Biol. sol, 1 (2) : 123-408.

Caplance (J.) et Laville (H.). 1968. - Etude morphométrique et physicochimique de neuf lacs du massif de Néouvielle (Hautes-Pyrénées). Annls Limnol., 4 (2) : 275-324.

Capblance (J.) et Laville (H.). 1972. - Etude de la productivité du lac de Port-Bielh (Pyrénées-Centrales). Proc. I.B.P. - U.N.E.S.C.O. Symposium on Productivity Problems of Freshwaters, KaziemierzDolny. Poland. May 6-12 1970 : 73-88. 
CaPBlance (J.). 1972. - Phytoplancton et productivité primaire de quelques lacs d'altitude dans les Pyrénées. Annls Limnol. 8 (3) : 231-321.

Edmondson (W. T.) et Winberg (G. G.). 1971. - A manual on methods for the assessment of secondary productivity in fresh waters. I.B.P. Handbook $\mathrm{n}^{\circ} 17$ Blackwell, Oxford and Edinburgh $358 \mathrm{p}$.

Fryer (G.). 1957. - The food of some freshwater Cyclopoid, Copepods and its ecological significance. J. Anim. Ecol., 26 : 263-286.

Giani (N.) et Laville (H.). 1973. - Le cycle biologique et la production de Sialis lutaria (Megaloptera) dans le lac de Port-Bielh (Pyrénées Centrales). Annls Limnol., 9 (1) : 45-61.

Laville (H.). 1971 a. - Recherches sur les Chironomides (Diptera) lacustres du massif de Néouvielle (Hautes-Pyrénées) : Systématique, Ecologie, Phénologie. Annls Limnol., 7 (2) : 173-332.

Laville (H.). 1971 b. - Recherches sur les Chironomides (Diptera) lacustres du massif de Néouvielle (Hautes-Pyrénées) : Communautés et Production. Annls Limnol., 7 (3) : 335-414.

Laville (H.) et Giani (N.). (sous presse). - Phénologie et cycles biologiques des Chironomides de la zone littorale $(0-7 \mathrm{~m})$ du lac de Port-Bielh (Pyrénées Centrales).

MACAN (T. T.). 1970. - Biological studies of the english lakes. Longman, London : $260 \mathrm{p}$.

Pacaud (A.). 1939. - Contribution à l'écologie des Cladocères. Bull. Biol. Fr. et Belg., Suppl. $25: 256$ p.

Rey (J.). 1968. - Ecologie des Cladocères du massif de Néouvielle Hautes-Pyrénées). Annls Limnol., 4 (3) : 325-356.

Rey (J.) et Capblance (J.). (en préparation). - Peuplement zooplanctonique d'un lac d'altitude des Pyrénées. 